\title{
Criminologie
}

\section{Le clan des Dubois}

\section{Alice Parizeau}

Volume 10, numéro 1, 1977

La criminalité des affaires au Québec

URI : https://id.erudit.org/iderudit/017068ar

DOI : https://doi.org/10.7202/017068ar

Aller au sommaire du numéro

Éditeur(s)

Les Presses de l'Université de Montréal

ISSN

0316-0041 (imprimé)

1492-1367 (numérique)

Découvrir la revue

Citer ce compte rendu

Parizeau, A. (1977). Compte rendu de [Le clan des Dubois]. Criminologie, 10(1), 94-97. https://doi.org/10.7202/017068ar

Ce document est protégé par la loi sur le droit d'auteur. L’utilisation des services d'Érudit (y compris la reproduction) est assujettie à sa politique d'utilisation que vous pouvez consulter en ligne.

https://apropos.erudit.org/fr/usagers/politique-dutilisation/
Cet article est diffusé et préservé par Érudit.

Érudit est un consortium interuniversitaire sans but lucratif composé de l’Université de Montréal, l’Université Laval et l'Université du Québec à Montréal. Il a pour mission la promotion et la valorisation de la recherche. https://www.erudit.org/fr/ 
Note de lecture

\section{LE CLAN DES DUBOIS}

Alice Parizeau

On sait que le crime organisé existe, que des règlements de comptes ont lieu dans la plupart des grandes villes nord-américaines et que des puissances occultes protègent les bandits qui exploitent quotidiennement de braves gens. Il n'en reste pas moins que le livre de Richard Desmarais* demeure un document unique en son genre. Il s'agit, en effet, d'une reconstitution de certains témoignages devant la Commission d'enquête sur le crime organisé, complétés par des extraits de rapports de police et des recoupements de divers articles de journaux.

A travers le livre, on retrace ainsi une sorte de carte de la ville de Montréal qui est celle des réseaux du crime organisé et de citoyens trop familiers de l'intimidation et de l'extorsion pour songer à faire appel aux forces de l'ordre. On réalise soudain que cette forme abjecte de contrainte n'est pas issue de l'imagination d'un scénariste américain, mais demeure une réalité qui se trouve à notre portée, à quelques rues de distance de notre lieu de travail ou d'habitation.

Ce qui surprend le plus cependant, ce n'est pas que cette contrainte existe, mais bien plus qu'elle est tolérée, enracinée et durable en quelque sorte à travers plusieurs années. Certes, les victimes paralysées par la peur se taisent, mais les protagonistes des drames sont connus de la police et continuent néanmoins de réaliser impunément des profits astronomiques. Tout se passe comme si les tueurs étaient non seulement protégés par le silence des témoins, mais aussi par des personnages puissants qui demeurent leurs très obéissants débiteurs. Au-delà des clans criminels se situent, en somme, des complicités qui remettent en cause le régime démocratique et jusqu'au principe même de la liberté d'élection des représentants du peuple.

Phénomène d'autant plus inquiétant que le livre relate des événements récents et qu'on ne peut éviter de se poser des ques-

* Richard Desmarais (1976), Le Clan des Dubois, Montréal, éd. internationales Alain Stanké. 
tions sur ce qui se passe dans l'actualité dont on traitera peutêtre un jour, puisque dans l'immédiat on craint un procès pour libelle diffamatoire, toujours possible, faute de preuves irréfutables.

* Le 20 juin 1956 », écrit Richard Desmarais, \& Saint-Henri fête la victoire du candidat libéral Philippe Lalonde. En dépit de l'heure tardive, les enfants errent encore dans les rues... Le flash illumine, l'instant d'un éclair, les traits des hommes placés dans l'ombre; au premier plan, Philippe Lalonde, le héros de la fête et Pit Lessard, qui sera député fédéral de Saint-Henri, deux ans plus tard. Albert Hamel, Henri Ouimet et Ralph Vichira, les organisateurs du parti, ont pris place à l'arrière : deux autres hommes se tiennent à l'extrême gauche de ce groupe : Normand Dubois et Alfred Quenneville. ,

Philippe Lalonde a été député provincial du comté de SaintHenri, de 1952 à 1968, sous l'étiquette libérale. En 1960, il a été adjoint parlementaire du Premier ministre, Jean Lesage, et il est décédé en 1974, à l'âge de 74 ans, soit il y a à peine deux ans. En ce qui a trait à Alfred Quenneville, il fut officier à la Sûreté du Québec et on l'avait congédié en 1950 , soit six ans avant l'élection décrite dans le livre, pour participation à l'impression de 60000 faux billets de loterie Irish Sweepstake. Normand Dubois, finalement déjà connu de la police ainsi que son père et ses frères, devait être appréhendé en 1957, pour vol avec violence et condamné à $\$ 200$ d'amende bien qu'il possédât un dossier judiciaire et qu'il ait été mêlé, peu avant, à un attentat « au cours duquel... on a rasé la tête d'une adolescente avant de l'exposer nue et solidement ligotée sur le parvis d'une église ».

Et tout au long du livre on retrouve des faits tout aussi surprenants que celui-là qui semblent témoigner d'une sorte d'impunité incompréhensible et troublante dont bénéficient des personnages pratiquant l'intimidation, les vols à main armée, les assauts et les meurtres.

Ce n'est pas l'appareil de la justice qui élimine le réseau du crime organisé, mais les règlements de comptes entre les divers individus ou groupes. "D'après ce que vous savez », demande-t-on à un témoin, « un individu comme Claude Dubeau, par exemple, combien peut-il avoir commis de meurtres ? > "Une dizaine... s répond l'homme interrogé et l'auteur du livre le rapporte sans ajouter aucun commentaire. 
En lisant l'ouvrage de Richard Desmarais, on constate donc que notre justice semble impuissante face au « milieu > invulnérable aussi longtemps que sa solidarité n'est pas remise en cause par une bande qui essaie de s'emparer d'un quartier et de déloger celle qui $\mathbf{y}$ règne en maître.

La dernière question qui se pose concerne les individus impliqués et leurs motivations. $\bar{A}$ travers les photos dont le livre est illustré, à travers les biographies sommaires, on retrouve la même constante. Une enfance malheureuse, une famille criminogène ou indifférente, des échecs scolaires et des réussites lors des premiers vols, puis l'engrenage et une carrière criminelle où il faut s'imposer comme partout ailleurs. L'image est classique tout en cessant d'être émouvante.

Contrairement aux légendes issues de certains récits et de certains films, il s'agit d'hommes laids, brutaux et immensément riches, qui ignorent non seulement les sentiments nobles, tels l'amour ou l'amitié, mais qui manquent également du plus élémentaire sens de la fantaisie.

Pour faire leurs affaires, ils tuent et passent ensuite leur temps à se protéger pour ne pas subir le même sort. Ils sont à la merci du plus petit des comparses et ne se déplacent qu'en compagnie de gardes du corps dont la fidélité, elle aussi, est souvent très relative.

$A$ en croire le récit de leurs aventures telles que relatées par Richard Desmarais, c'est un univers d'hommes où les femmes sont rares et où elles assument le rôle d'entraîneuses, mais jamais celui de « reines du milieu ». Le matriarcat n'a pas cours ici et seuls les hommes s'imposent par la force. Le lecteur n'éprouve aucune sympathie en lisant l'histoire des frères Dubois, ni aucune envie de les connaître. Le livre ne suscite, dans son ensemble, qu'un étonnement indigné ou encore un goût de répression parfaitement antidémocratique et contraire aux principes mêmes d'une justice qui se veut civilisée. C'est là en somme, la principale lacune du document qu'est cet ouvrage. $\AA$ travers les faits rapportés dans toute leur nudité, on cesse de considérer les criminels comme des êtres humains et on les assimile à des machines à tuer dont la précision et l'aveuglement sont parfaitement répugnants.

En ce qui a trait aux victimes, par contre, un certain groupe ne semble être «coupable », ni de légèreté, ni d'insouciance. Les 
vraies victimes, ce sont des gens qui habitent dans les quartiers devenus des zones grises de la grande ville où des enfants jouent dans les rues sales et où les adultes se contentent de survivre, trop épuisés par leur condition socio-économique et culturelle pour essayer de vivre. La deuxième catégorie des victimes comprend les anonymes, soit ceux qui jouent dans des tripots, fréquentent des boîtes de nuit et d'une façon plus générale profitent de leur propre gré des services que la pègre s'empresse de leur offrir. Mais même là, on constate que l'ignorance prédomine sur le goût de l'aventure ou du risque. En fait tout se passe le plus simplement du monde. Des hommes et des femmes dansent dans des boites de nuit de Montréal sans soupçonner un instant que le serveur qui leur apporte les consommations est en fait un tueur à gages, tandis que le patron qui vient à l'occasion les saluer n'est qu'un otage d'un " groupe » qui fait des affaires à ses dépens. La troisième catégorie des victimes ne suscite, elle, aucune sympathie, car ce sont les membres du crime organisé qu'on élimine à la suite d'une erreur de jugement ou d'une "malchance s.

Que conclure à partir de tout cela ? Comment réagir ? Doiton devenir une société puritaine dans le plus profond sens de ce terme pour éviter de faire le jeu de la pègre et du crime organisé ? Cela est-il réalisable?

Nous ne le croyons pas, puisque l'histoire démontre que le puritanisme n'a jamais permis à aucune société d'eliminer la criminalité. Ce qui est certain cependant, c'est qu'il est urgent de dépister et de réprimer toutes les formes de l'alliance entre les autorités publiques et syndicales et les mondes mystérieux du crime organisé et d'ajuster constamment des mécanismes de contrôle de façon à ce que la justice ne soit pas paralysée par des pactes inavouables signés pour services rendus à la suite d'une élection, d'une manifestation ou d'une autre action socio-politique faisant partie, en tant que telle, du processus normal d'un régime qui se veut démocratique. 